\title{
Miranda
}

Revue pluridisciplinaire du monde anglophone /

Multidisciplinary peer-reviewed journal on the English-

speaking world

$12 \mid 2016$

Mapping gender. Old images ; new figures

\section{Laurent Coq / Walter Smith III, The Lafayette Suite.} Laurent Coq (piano), Walter Smith III (saxophone ténor), Joe Sanders (contrebasse et basse électrique), Damion Reid (batterie).

CD publié sur le label Jazz \& People et enregistré à Tedesco Studios (New Jersey), les $1^{\mathrm{e}}$ et 2 décembre 2014

\section{Ludovic Florin}

\section{(2) OpenEdition}

\section{Journals}

Édition électronique

URL : http://journals.openedition.org/miranda/8475

DOI : $10.4000 /$ miranda.8475

ISSN : 2108-6559

Éditeur

Université Toulouse - Jean Jaurès

Référence électronique

Ludovic Florin, «Laurent Coq / Walter Smith III, The Lafayette Suite. », Miranda [En ligne], 12 | 2016, mis en ligne le 01 mars 2016, consulté le 16 février 2021. URL : http://journals.openedition.org/miranda/ 8475 ; DOI : https://doi.org/10.4000/miranda.8475

Ce document a été généré automatiquement le 16 février 2021.

\section{cc) (1)}

Miranda is licensed under a Creative Commons Attribution-NonCommercial-NoDerivatives 4.0 International License. 


\title{
Laurent Coq / Walter Smith III, The Lafayette Suite.
}

\author{
Laurent Coq (piano), Walter Smith III (saxophone ténor), Joe Sanders \\ (contrebasse et basse électrique), Damion Reid (batterie). \\ CD publié sur le label Jazz \& People et enregistré à Tedesco Studios (New \\ Jersey), les $1^{\mathrm{e}}$ et 2 décembre 2014
}

\section{Ludovic Florin}

1 Tout le monde connaît Lafayette, et pas seulement parce que les villes de France et de Navarre possèdent toutes, ou presque, un boulevard, une rue, une impasse à son nom. Jeune aristocrate porté par des idéaux forts, il décide, contre l'avis de son roi, de traverser l'Atlantique pour se battre aux côtés des Américains dans la Guerre d'indépendance qui les opposent au pouvoir colonial britannique. La victoire de Yorktown en octobre 1781, dont il fut l'un des artisans, en plus de ses activités sur le sol d'outre-Atlantique, lui valent d'être devenu l'un des huit citoyens d'honneur des ÉtatsUnis d'Amérique en 2002. En France, comme parlementaire, il présenta le 11 juillet 1789 un projet de Déclaration des droits de l'homme et du citoyen. Bien que non retenue, cette Déclaration anticipait celle qui allait être bientôt adoptée. En octobre de la même année, il soumet à l'Assemblée Constituante les droits civils des hommes de couleur, la suppression des ordres monastiques, l'abolition de la noblesse héréditaire, l'égalité des citoyens. Il n'est guère étonnant, dès lors, que ce personnage de roman, mort après la Monarchie de Juillet à l'issue d'une vie bien remplie, ait inspiré deux musiciens de jazz, l'un français, l'autre américain, tous deux apôtres de la communion des peuples, comme le fut Lafayette. Certes, l'histoire du champ jazzistique est marquée, de loin en loin, d'hommages rendus à Gilbert du Motier de La Fayette : Bennie Moten en 1932, Hot Lips Page en 1940, Bruce Turner en 1962, Derek Bailey en 1995... Mais Laurent Coq et Walter Smith III sont les premiers à lui avoir consacré tout un album.

2 La création de ce dernier aura été possible grâce notamment à un prestigieux programme franco-américain qui associe la fondation FACE (French American Culture Exchange, financée par l'Institut Français) et la MAAF (Mid Atlantic Arts Fondation) basée à Baltimore. Chaque année, une bourse assez conséquente est accordée à cinq - 
parfois sept - projets qui associent musiciens français et américains. Lauréats du concours en 2013, Laurent Coq et Walter Smith III (prononcer "the third») se sont donnés un an pour, d'une part, trouver les quelques fonds encore manquants afin de boucler le budget attaché à l'enregistrement et pour, d'autre part, s'accorder le temps de travailler la musique après son écriture, de se familiariser avec elle afin de pouvoir se sentir libres et prendre le large une fois entrés en studio. Pour ce qui est du budget, faut-il s'étonner que les Galeries Lafayette aient refusé de participer au financement de la réalisation de cet album ? De ce fait, les deux artistes ont eu recours à la générosité de donateurs, en faisant appel au don en ligne, via le site KissKissBankBank. Cent vingttrois mélomanes ont ainsi permis à ce disque de voir finalement le jour.

Laurent Coq est bien connu des mélomanes français attachés au jazz. Depuis ses débuts (album Jaywalker de 1997), il s'est fait remarquer par sa faculté à rester dans l'idiome du jazz tout en en prolongeant l'expression. Très rapidement, il a joué avec des musiciens américains de passage sur le sol français, mais il est aussi, dès 1994, parti pour New York afin de se perfectionner avec certains d'entre eux. Depuis, tout en affirmant sa singularité, il n'a cessé d'entretenir des relations fécondes avec quelques-unes des figures marquantes du jazz contemporain américain, tels que le batteur de la Lafayette Suite, Damion Reid (qu'il connaît depuis 2001), aujourd'hui considéré comme l'un des régénérateurs de la pratique batteristique actuelle. La collaboration de Laurent Coq avec Walter Smith III (le saxophoniste du quintette du trompettiste Ambrose Akinmusire) s'inscrit en toute logique dans le parcours artistique du pianiste. Comme l'altiste de haut niveau Miguel Zenon avec qui Laurent Coq a réalisé Rayuela (en 2011, à partir de textes de Julio Cortázar), ils sont d'abord tous deux de remarquables mélodistes, dimension musicale à laquelle ils ne veulent ni ne peuvent renoncer. D'un même élan, ils aiment aussi interroger les métriques les plus complexes, en faisant toujours en sorte que cela ne paraisse jamais abstrus. Et lorsque le quartette use de la vieille mesure à trois temps, comme sur « Major Benjamin Huger », c'est d'une manière tout à fait contemporaine : sans jamais dissoudre ce rebondi si caractéristique du jazz, la métrique n'apparaît pourtant qu'en filigrane, à la fois ni appuyée, ni dissolue. À cet égard, Walter Smith III reste fidèle aux préceptes esthétiques présents dès son premier album, Casually Introducing (2006), dont le graphisme décalquait celui de Fushia Swing Song (Blue Note, 1964) de Sam Rivers, saxophoniste dont les fondements stylistiques restèrent ancrés dans le hardbop, mais en tenant compte des aspects libertaires du free jazz. Il convient donc de ne pas se tromper au sujet du quartette Coq-Smith : ainsi, il serait déplacé de les qualifier de "néo-boppers" (un courant porté par Wynton Marsalis dans les années 1980), car à aucun moment ils n'aspirent à rejouer le jazz du temps passé. Fidèles à l'esprit de cette musique, ils continuent de la faire progresser tout en continuant de porter certaines valeurs qu'ils considèrent comme fondamentales (le swing, donc, mais aussi l'interaction, le son individuel au profit d'un son de groupe, le lien entre écriture et improvisation, etc.).

Comme le titre même le suggère, dans The Lafayette Suite les plages s'enchaînent les unes aux autres, sans aucun arrêt. Chaque mouvement de ce tone poem évoque les exploits américains de Lafayette, les plages paires étant de la plume de Laurent Coq et les impaires de Walter Smith III. Rappelons que si en musique "classique » on traduit tone poem par "poème symphonique " - c'est-à-dire une composition orchestrale inspirée par une trame extra-musicale que la musique seule s'efforce de narrer -, en jazz ces mots renvoient à une sorte de ballade typique du Second Quintette de Miles Davis (1964-1968), à l'ambiance singulière fortement marquée et à la structure élaborée 
(peu ou pas de reprises de parties). Bien que l'album ne soit, bien sûr, pas uniquement constitué de ballades, la dimension fortement évocatrice attachée à l'expression tone poem pourrait parfaitement convenir à The Lafayette Suite.

Quelle que soit la dénomination assignée à cette œuvre, l'ambition est claire : faire en sorte que les presque cinquante minutes de musique coulent de la manière la plus naturelle, la plus organique possible, sans que jamais l'attention de l'auditeur ne soit saturée, ou qu'il finisse par s'ennuyer. D'emblée, les musiciens aspirent ainsi aux plus hautes réussites de cette musique populaire-savante qu'est le jazz, incarnée par les compositions de Duke Ellington, André Hodeir, Dave Brubeck, et de bien d'autres encore. À l'image de la collaboration entre le Duke et son double Billy Strayhorn, il n'est ici pas souvent possible de déterminer «à l'aveugle " le moment où l'on passe d'un compositeur à l'autre. C'est dire combien cette réalisation à deux plumes se révèle cohérente et homogène.

6 Affirmons-le dès lors sans détour : on prend beaucoup de plaisir à l'écoute de ce disque. Comme chacun de ses projets, Laurent Coq frappe d'un sceau unique cette nouvelle création originale. Sa réflexion sur la forme, par exemple, est tout à fait remarquable. Depuis ses débuts, le jazz s'interroge sur la manière dont il est possible de sortir de la sempiternelle structure thème-improvisation-thème. Il semble que les deux compositeurs soient parvenus à une solution artistique des plus remarquables : outre la forme suite, chaque pièce propose en effet un développement chaque fois nouveau, renouvelant constamment l'intérêt. L'orchestration en lien avec les modes de jeu est une autre source de satisfaction pour le mélomane qui saura apprécier la palette étonnamment étendue de ce «simple» quartette (voir par exemple la coda de «Charleston, South Carolina, June 13, 1777 »). Parfois délaissée par les jazzmen, la dimension contrapuntique (plusieurs lignes mélodiques indépendantes formant un tout), qu'elle soit improvisée ou écrite, a particulièrement été soignée, ce qui renforce la (haute) qualité générale de l'ouvrage et autorise de multiples réécoutes attentives.

7 In fine, les notions portées haut par Lafayette se trouvent ici incarnées : émancipation, liberté, indépendance... Elles constituent le sang vif d'une musique digne de son époque, la nôtre.

\section{INDEX}

Thèmes : Music

Keywords : composition, improvisation, jazz, orchestration, quartet, swing, tone poem

Mots-clés : composition, improvisation, jazz, orchestration, quartette, swing, tone poem 


\section{AUTEURS}

\section{LUDOVIC FLORIN}

Maître de Conférences

Université Toulouse-Jean Jaurès

ludovic.florin@wanadoo.fr 